\title{
2-GENERATION OF SIMPLE LIE ALGEBRAS AND FREE DENSE SUBGROUPS OF ALGEBRAIC GROUPS
}

\author{
ALLA S. DETINKO AND WILLEM A. DE GRAAF
}

\begin{abstract}
We construct generating pairs of simple Lie algebras in characteristic zero. We apply this construction to exhibit infinite series of 2-generator Zariski dense subgroups that are free of rank 2 of the simple algebraic groups $\operatorname{SL}(n, \mathbb{C}), \operatorname{Sp}(n, \mathbb{C}), G_{2}(\mathbb{C})$.
\end{abstract}

\section{INTRODUCTION}

The Tits alternative implies that each non-solvable-by-finite linear group $H$ in zero characteristic contains a free non-abelian subgroup [20, p. 145]. Moreover free subgroups are ubiquitous in $H$ (see, e.g., [1, 9, 11]). These subgroups are important for investigation of structure and properties of $H$, especially if a free subgroup is reasonably 'large', i.e. Zariski-dense in the closure of $H$; see, for examples, [5, 17]. Despite ubiquity, explicit construction of free dense subgroups is still a problem. A reason is that justification of freeness of a linear group in general is difficult (although it could be tested computationally whether a finitely generated linear group contains a free non-abelian subgroup, [8]). A famous example is the problem of freeness of the group

$$
H_{2}(t, s):=\left\langle\left(\begin{array}{cc}
1 & t \\
0 & 1
\end{array}\right),\left(\begin{array}{cc}
1 & 0 \\
s & 1
\end{array}\right)\right\rangle .
$$

Since the 1950s it is known that the group is free for $|t| \geq 2,|s| \geq 2$ as well as for $t, s$ algebraically independent over $\mathbb{Q}$; here $t, s \in \mathbb{C}$ ([18, p. 168], [20, p. 3032]). Nevertheless, up to now it is not known whether there are rational values of $t, s$ with $-2<t, s<2$ for which $H_{2}(t, s)$ is free; non-freeness has been justified for a range of such pairs (see, e.g., [3]).

In this paper we provide a method to construct free dense subgroups of rank 2 of simple algebraic groups. Using the method we obtain infinite series of rank 2 free dense subgroups in a number of simple algebraic groups, including $\operatorname{SL}(n, \mathbb{C})$, $\operatorname{Sp}(n, \mathbb{C})$. These free subgroups are produced in a similar way as $H_{2}(t, s)$ and are given by explicit generating sets of size 2 . In particular, we exhibit 2-parameter families of rank 2 free dense subgroups of $\operatorname{SL}(n, \mathbb{Z}), \operatorname{Sp}(n, \mathbb{Z})$, i.e. explicit examples of thin matrix groups (dense subgroups of infinite index [19]). Although thin subgroups are known to be ubiquitous, explicit examples still are rare [10, 11, 19]; see the recent paper [2] for a series of free dense subgroups in $\operatorname{SL}(n, \mathbb{C})$.

Our approach is based on the construction of 2-generator sets of simple Lie algebras which is of independent interest (cf. [14], [17, Section 2], [6]). If two nilpotent elements $x, y$ of the Lie algebra $\mathfrak{g}$ of a simple algebraic group $G$ generate $\mathfrak{g}$, then 
the group generated by the two elements $\exp (s x), \exp (t y)$ is Zariski-dense in $G$ (see Proposition 3.1). So the density of the group is guaranteed. We also manage to show that the groups are free for many values of $s, t$ by applying the so-called ping-pong lemma. In order to be able to apply this lemma we need generators $x, y$ that are sufficiently "nice" (i.e., seen as $n \times n$-matrices which have very few nonzero entries, allowing for a nice description of the exponentials). In the next section we go into the problem of finding such "nice" generators of a simple Lie algebra $\mathfrak{g}$. Then in Section 3 we apply it to obtain 2-generator free dense subgroups of rank 2 of simple algebraic groups.

\section{2-GENERATION OF SIMPLE LIE ALGEBRAS}

It is known that a split semisimple Lie algebra over a field of characteristic 0 has two elements generating it ([14], [17]). Here we consider the problem to find two nilpotent elements that generate a given semisimple Lie algebra. For our applications we want these generators to be as "nice" as possible, that is, viewing the Lie algebra as a matrix algebra, we want generators that have few nonzero coefficients, so that we have good control over their exponentials.

First we show that it is straightforward to find two nilpotent elements generating a given semisimple Lie algebra (Proposition 2.2). Subsequently we consider the Lie algebras $\mathfrak{s l}(n, K), \mathfrak{s o}(n, K)$ for $n$ odd and different from 7 and $\mathfrak{s p}(n, K)$, where $n$ is even. For each of these we find two "nice" nilpotent elements generating the Lie algebra. Along the way we also find a "nice" generating set of the simple Lie algebra of type $G_{2}$ in its realization as a subalgebra of $\mathfrak{s l}(7, K)$. In the end we prove a theorem on a subalgebra of a simple Lie algebra generated by two specific nilpotent elements (Theorem 2.8). This theorem plays no role in our paper, but we note it as a curiosity that we can prove using the previous results.

In our proofs we sometimes verify isolated cases by direct computation in the computer algebra system GAP4 ([12]).

The ground field $K$ is of characteristic 0 . We consider split semisimple Lie algebras $\mathfrak{g}$ over $K$. Alternatively, we could just consider semisimple Lie algebras over $\mathbb{C}$. Let $\mathfrak{h}$ be a Cartan subalgebra of $\mathfrak{g}$. Corresponding to that $\mathfrak{g}$ has a root system $\Phi$, with set of simple roots $\Delta=\left\{\alpha_{1}, \ldots, \alpha_{\ell}\right\}$ and Cartan matrix $C$. Then $\mathfrak{g}$ is generated by a canonical set of generators: $h_{i}, x_{\alpha_{i}} \in \mathfrak{g}_{\alpha_{i}}, x_{-\alpha_{i}} \in \mathfrak{g}_{-\alpha_{i}}$ for $1 \leq i \leq \ell$. These satisfy the relations (see [15], $\S$ IV.3)

$$
\begin{aligned}
{\left[h_{i}, h_{j}\right] } & =0 \\
{\left[h_{i}, x_{\alpha_{j}}\right] } & =C(j, i) x_{\alpha_{j}} \\
{\left[h_{i}, x_{-\alpha_{j}}\right] } & =-C(j, i) x_{-\alpha_{j}} \\
{\left[x_{\alpha_{i}}, x_{-\alpha_{j}}\right] } & =\delta_{i, j} h_{i} .
\end{aligned}
$$

Proposition 2.1. Let $h \in \mathfrak{h}$ be such that $\left\{ \pm \alpha_{i}(h) \mid 1 \leq i \leq \ell\right\}$ consists of $2 \ell$ distinct elements. Let $u=\sum_{j=1}^{\ell} a_{i} x_{\alpha_{i}}+\sum_{i=1}^{\ell} b_{i} x_{-\alpha_{i}}$, with all $a_{i}, b_{i}$ nonzero. Then $h, u$ generate $\mathfrak{g}$. 
Proof. Let $m=2 \ell$ and $\beta_{1}, \ldots, \beta_{m}=\alpha_{1}, \ldots, \alpha_{\ell},-\alpha_{1}, \ldots,-\alpha_{\ell}$. Then $u=$ $\sum_{j} c_{j} x_{\beta_{j}}$. Define $\left[h^{0}, u\right]=u,\left[h^{i+1}, u\right]=\left[h,\left[h^{i}, u\right]\right]$. Then $\left[h^{i}, u\right]=\sum_{j} \beta_{j}(h)^{i} c_{j} x_{\beta_{j}}$. So if we consider the elements $\left[h^{i}, u\right]$ for $0 \leq i \leq m-1$, put their coefficient vectors into a matrix then we get a Vandermonde matrix so that the determinant is

$$
c_{1} \cdots c_{m} \prod_{i<j}\left(\beta_{j}(h)-\beta_{i}(h)\right)
$$

Hence it is nonzero, so that the space spanned by the $\left[h^{i}, u\right]$ contains all $x_{\alpha_{j}}$. Therefore, $h, u$ generate $\mathfrak{g}$.

Proposition 2.2. Let $x=x_{\alpha_{1}}+\cdots+x_{\alpha_{\ell}}, y=b_{1} x_{-\alpha_{1}}+\cdots+b_{\ell} x_{-\alpha_{\ell}}, b_{i} \neq 0$ for all $i$. Let $b \in K^{\ell}$ be the vector with coordinates $b_{i}$. Write $v_{1}, \ldots, v_{\ell}$ for the coordinates of $C b$. Suppose that $\left\{ \pm v_{i} \mid 1 \leq i \leq \ell\right\}$ consists of $2 \ell$ distinct elements.

Then $x, y$ generate $\mathfrak{g}$.

Proof. Let $h=[x, y]$ then $h=b_{1} h_{1}+\cdots+b_{\ell} h_{\ell}$ and $\alpha_{i}(h)=v_{i}$. So we get the conclusion from Proposition 2.1 by setting $u=x+y$.

Example 2.3. Let the root system be of type $A_{\ell}$, with the standard ordering of simple roots. For $1 \leq i \leq \ell$ let $b_{i}=\sum_{j=1}^{i} 2^{n-j}$. Let $x=x_{\alpha_{1}}+\cdots+x_{\alpha_{\ell}}$, $y=b_{1} x_{-\alpha_{1}}+\cdots+b_{\ell} x_{-\alpha_{\ell}}$. Then $x, y$ generate $\mathfrak{g}$.

Indeed, let $v$ be as in Proposition 2.2. Then $v_{1}, \ldots, v_{\ell}=2^{\ell-2}, 2^{\ell-3}, \ldots, 2,1,2^{\ell}$. So the result follows.

Let $e_{i, j}$ denote the $n \times n$-matrix with a 1 on position $(i, j)$ and zeros elsewhere. For $x, y \in \mathfrak{g l}(n, K)$ we define $\left[x^{0}, y\right]=y$ and $\left[x^{s+1}, y\right]=\left[x,\left[x^{s}, y\right]\right]$ for $s \geq 0$. Also we use the following result, which is well-known, and not difficult to prove.

Lemma 2.4. Let $\mathfrak{g}$ be a split simple Lie algebra over $K$, with canonical generators $h_{i}, x_{\alpha_{i}}, x_{-\alpha_{i}}, 1 \leq i \leq \ell$. Let $\alpha_{0}$ be the highest root of the root system of $\mathfrak{g}$, and $y_{0} \in \mathfrak{g}_{-\alpha_{0}}, y_{0} \neq 0$. Then $\mathfrak{g}$ is generated by $\left\{x_{\alpha_{1}}, \ldots, x_{\alpha_{\ell}}, y_{0}\right\}$.

The next proposition has also been proved in [6] for $n$ odd.

Proposition 2.5. Let $n \geq 3$ and $x=\sum_{i=1}^{n-1} e_{i, i+1}, y=e_{n, 1}$. Let $\mathfrak{g}$ denote the Lie algebra generated by $x, y$. Then $\mathfrak{g}$ is simple. If $n=2 m$ is even then $\mathfrak{g}$ is of type $C_{m}$. If $n$ is odd, then $\mathfrak{g}$ is of type $A_{n-1}$.

Proof. By induction on $s$ we have

$$
\left[x^{s}, y\right]=\sum_{i=\max (0, s-n+1)}^{\min (s, n-1)}(-1)^{i}\left(\begin{array}{l}
s \\
i
\end{array}\right) e_{n-s+i, i+1}
$$


Let $1 \leq r \leq\left\lfloor\frac{n}{2}\right\rfloor$ and $u=\left[x^{2 n-1-r}, y\right], v=\left[x^{r-1}, y\right]$ then

$$
\begin{aligned}
& u=\sum_{i=n-r}^{n-1}(-1)^{i}\left(\begin{array}{c}
2 n-1-r \\
i
\end{array}\right) e_{r+1-n+j, j+1} \\
& v=\sum_{i=0}^{r-1}(-1)^{i}\left(\begin{array}{c}
r-1 \\
i
\end{array}\right) e_{n-r-1-i, i+1}
\end{aligned}
$$

so after some manipulation we obtain

$$
[u, v]=(-1)^{n-r} \sum_{k=1}^{r}\left(\begin{array}{c}
r-1 \\
k-1
\end{array}\right)\left(\begin{array}{c}
2 n-r-1 \\
n-r-1+k
\end{array}\right)\left(e_{k, k}-e_{n-r+k, n-r+k}\right) .
$$

Considering this for $r=1,2, \ldots$ we see that $e_{r, r}-e_{n+1-r, n+1-r} \in \mathfrak{g}$ for $1 \leq r \leq$ $\left\lfloor\frac{n}{2}\right\rfloor$. Now $\left[e_{1,1}-e_{n, n}, x\right]=e_{1,2}+e_{n-1, n}$ and for $r \geq 2$

$$
\left[e_{r, r}-e_{n+1-r, n+1-r}, x\right]=e_{r, r+1}+e_{n-r, n-r+1}-e_{r-1, r}-e_{n+1-r, n+2-r},
$$

from which it follows that $e_{r, r+1}+e_{n-r, n-r+1} \in \mathfrak{g}$ for $1 \leq r<\frac{n}{2}$.

Now suppose that $n=2 m$. Let $h_{0}=\left[x^{n-1}, y\right]=\sum_{l=1}^{n}(-1)^{l-1}\left(\begin{array}{c}n-1 \\ l-1\end{array}\right) e_{l, l}$. Then $\left[h_{0}, x\right]=\sum_{k=1}^{n-1}(-1)^{k-1}\left(\begin{array}{l}n \\ k\end{array}\right) e_{k, k+1}$. But this is equal to

$$
\sum_{k=1}^{m-1}(-1)^{k-1}\left(\begin{array}{l}
n \\
k
\end{array}\right)\left(e_{k, k+1}+e_{n-k, n-k+1}\right)+(-1)^{m-1}\left(\begin{array}{l}
n \\
m
\end{array}\right) e_{m, m+1} .
$$

So it follows that $e_{m, m+1} \in \mathfrak{g}$.

Define the linear map $\varphi: \mathfrak{g l}(n, K) \rightarrow \mathfrak{g l}(n, K)$ by $\varphi\left(e_{i, j}\right)=(-1)^{i-j+1} e_{n-j+1, n-i+1}$. Then $\varphi$ is an automorphism of $\mathfrak{g l}(n, K)$, leaving invariant $\mathfrak{s l}(n, K)$. So its restriction to the latter is an automorphism of $\mathfrak{s l}(n, K)$. By inspection it is seen that $\varphi$ is equal to the nontrivial diagram automorphism of $\mathfrak{s l}(n, K)$. Let $\mathfrak{a}=$ $\{z \in \mathfrak{s l}(n, K) \mid \varphi(z)=z\}$. Then it is known that $\mathfrak{a}$ is a simple Lie algebra of type $C_{m}$ (see, for example, [16], §7.9). Since $x, y \in \mathfrak{a}$ we have $\mathfrak{g} \subset \mathfrak{a}$. For $1 \leq i \leq m-1$ set $x_{i}=e_{i, i+1}+e_{n-i, n-i+1}, y_{i}=e_{i+1, i}+e_{n-i+1, n-i}$, $h_{i}=e_{i, i}-e_{i+1, i+1}+e_{n-i, n-i}-e_{n-i+1, n-i+1}$ and $x_{m}=e_{m, m+1}, y_{m}=e_{m+1, m}$, $h_{m}=e_{m, m}-e_{m+1, m+1}$. Then these elements satisfy the relations of a canonical generating set of a Lie algebra of type $C_{m}$. Hence they generate a subalgebra of $\mathfrak{a}$ of type $C_{m}$ (see for example, [7]). But they all lie in $\mathfrak{a}$, so it follows that they generate $\mathfrak{a}$. We have that $y$ is the lowest root vector of $\mathfrak{a}$. So $\mathfrak{a}$ is also generated by the $x_{i}$ along with $y$ (Lemma 2.4). But all these elements lie in $\mathfrak{g}$. The conclusion is that $\mathfrak{g}=\mathfrak{a}$.

Now suppose that $n$ is odd. Again we have the element $h_{0}=\sum_{l=1}^{n}(-1)^{l-1}\left(\begin{array}{c}n-1 \\ l-1\end{array}\right) e_{l, l}$ in $\mathfrak{g}$. Also $x_{i}=e_{i, i+1}+e_{n-i, n-i+1} \in \mathfrak{g}$ for $1 \leq i \leq \frac{n-1}{2}$. A short calculation shows that

$$
\left[h_{0}, x_{i}\right]=(-1)^{i}\left(\begin{array}{c}
n \\
i
\end{array}\right)\left(-e_{i, i+1}+e_{n-i, n-i+1}\right) .
$$


Hence $e_{i, i+1} \in \mathfrak{g}$ for $1 \leq i \leq n-1$. These are the simple root vectors of $\mathfrak{s l}(n, K)$. Again, $y$ is a lowest root vector of $\mathfrak{s l}(n, K)$. It follows that $\mathfrak{g}=\mathfrak{s l}(n, K)$.

Proposition 2.6. Let $n \geq 4$ be even, $x=\sum_{i=1}^{n-1} e_{i, i+1}, y=e_{n-1,1}+e_{n, 2}$. Let $\mathfrak{g}$ denote the Lie algebra generated by $x, y$. Then $\mathfrak{g}=\mathfrak{s l}(n, K)$.

Proof. For integers $s \geq 0$ and $i$ define $C(s, i)=\left(\begin{array}{c}s \\ i\end{array}\right)-\left(\begin{array}{c}s \\ i-1\end{array}\right)$. So $C(s, 0)=$ $1, C(s, s+1)=-1$ and $C(s, i)=0$ if $i<0$ or $i>s+1$. Furthermore, $C(s, i)+C(s, i-1)=C(s+1, i)$.

By induction on $s$ it follows that

$$
\left[x^{s}, y\right]=\sum_{i=\max (0, s-n+2)}^{\min (s+1, n-1)}(-1)^{i} C(s, i) e_{n-s+i-1, i+1} .
$$

Set $h_{0}=\left[x^{n-2}, y\right]$ then

$$
h_{0}=\sum_{i=0}^{n-1}(-1)^{i} C(n-2, i) e_{i+1, i+1} .
$$

Then $\left[h_{0}, y\right]=(n-2)\left(-e_{n-1,1}+e_{n, 2}\right)$. Hence $z=e_{n-1,1} \in \mathfrak{g}$. Furthermore we set

$$
w=\left[x^{n-3}, y\right]=\sum_{i=0}^{n-2}(-1)^{i} C(n-3, i) e_{i+2, i+1} .
$$

Then $[w, z]=C(n-3, n-2) e_{n, 1}$. Hence $e_{n, 1} \in \mathfrak{g}$. So from the proof of Proposition 2.5 it follows that $e_{r, r+1}+e_{n-r, n-r+1} \in \mathfrak{g}$ for $1 \leq r<\frac{n}{2}$. Using the hypothesis that $n$ is even we compute

$$
\begin{aligned}
{\left[h_{0}, e_{r, r+1}+e_{n-r, n-r+1}\right] } & =(-1)^{r-1}\left(C(n-1, r) e_{r, r+1}+C(n-1, n-r) e_{n-r, n-r+1}\right) \\
& =(-1)^{r-1} C(n-1, r)\left(e_{r, r+1}-e_{n-r, n-r+1}\right) .
\end{aligned}
$$

It follows that $e_{r, r+1}, e_{n-r, n-r+1} \in \mathfrak{g}$ for $1 \leq r<\frac{n}{2}$. (Note that $C(n-1, r) \neq 0$ for $r<\frac{n}{2}$.) In the proof of Proposition 2.5 we have also seen that $e_{m, m+1} \in \mathfrak{g}$, where $n=2 m$. By Lemma2.4 it now follows that $\mathfrak{g}=\mathfrak{s l}(n, K)$.

Proposition 2.7. Let $n=2 m+1 \geq 5$ be odd, $x=\sum_{i=1}^{n-1} e_{i, i+1}, y=e_{n-1,1}+e_{n, 2}$. Let $\mathfrak{g}$ denote the Lie algebra generated by $x, y$. Then $\mathfrak{g}=\mathfrak{s o}(n, K)$ if $n \neq 7$ and $\mathfrak{g}$ is simple of type $G_{2}$ if $n=7$.

Proof. The proof for $n=7$ is a direct computation with GAP. So in the remainder of the proof we assume $n \neq 7$.

We consider the diagram automorphism $\varphi$ as in the proof of Proposition 2.5 . Since $n$ is odd it leaves the generators $x, y$ invariant. It is known that $\mathfrak{a}=\{z \in$ $\mathfrak{s l}(n, K) \mid \varphi(z)=z\}$ is a simple Lie algebra of type $B_{m}$ (see [16] $\left.\S 7.9\right)$. Hence $\mathfrak{a}$ is $\mathfrak{s o}(n, K)$ and $\mathfrak{g} \subset \mathfrak{s o}(n, K)$.

Also, $y$ is a root vector of $\mathfrak{s o}(n, K)$ corresponding to the lowest root. Simple root vectors are $x_{i}=e_{i, i+1}+e_{n+1-i, n+2-i}$ for $1 \leq i \leq m$. We show that $x_{i} \in \mathfrak{g}$ for $1 \leq i \leq m$. By Lemma 2.4 this finishes the proof. 
We have the same formula for $\left[x^{s}, y\right]$ as in the proof of the previous lemma. For $s=2$ that gives $\left[x^{2}, y\right]=e_{n-3,1}-e_{n-2,2}-e_{n-1,3}+e_{n, 4}$. Again set $h_{0}=\left[x^{n-2}, y\right]$, then using the same expression for $h_{0}$ as in the proof of the previous lemma, and some manipulation,

$\left[h_{0},\left[x^{2}, y\right]\right]=(C(n-2,3)-1)\left(e_{n-3,1}+e_{n, 4}\right)+(C(n-2,2)-C(n-2,1))\left(e_{n-2,2}+e_{n-1,3}\right)$.

If $n=5$ these coefficients are $-3,-2$, whereas for $n \geq 9$ they are both positive. Together with the expression for $\left[x^{2}, y\right]$ this shows that $e_{n-3,1}+e_{n, 4}$ and $e_{n-2,2}+$ $e_{n-1,3}$ lie in $\mathfrak{g}$. (For $n=7$ the coefficients are $-1,1$ and we see that the proof goes wrong here; unsurprising, as in this case the resulting Lie algebra is of type $G_{2}$.)

We have $\left[x^{2 n-4}, y\right]=-C(2 n-4, n-2)\left(e_{1, n-1}+e_{2, n}\right)$ so that $e_{1, n-1}+e_{2, n} \in \mathfrak{g}$. Furthermore, $\left[e_{1, n-1}+e_{2, n}, e_{n-2,2}+e_{n-1,3}\right]=e_{1,3}-e_{n-2, n}$. It follows that $e_{1,3}-e_{n-2, n} \in \mathfrak{g}$. Set $z=\left[x^{n-3}, y\right]$ then

$$
z=\sum_{j=1}^{n-1}(-1)^{j-1} C(n-3, j-1) e_{j+1, j} .
$$

Now $\left[z, e_{1,3}-e_{n-2, n}\right]=e_{2,3}+e_{n-2,1}+(n-4)\left(e_{1,2}+e_{n-1, n}\right)$.

For $0 \leq r \leq m-2$ set $u=\left[x^{2 n-4-r}, y\right], v=\left[x^{r}, y\right]$. Then

$$
\begin{aligned}
& u=\sum_{j=1}^{r+2}(-1)^{n-r-3+j} C(2 n-4-r, n-r-3+j) e_{j, n-r-2+j} \\
& v=\sum_{j=1}^{r+2}(-1)^{j-1} C(r, j-1) e_{n-r+j-2, j} .
\end{aligned}
$$

Then

$[u, v]=(-1)^{n-r} \sum_{j=1}^{r+2} C(r, j-1) C(2 n-4-r, n-3-r+j)\left(e_{j, j}-e_{n+1-j, n+1-j}\right)$.

Write $\gamma_{n, r}(j)=C(r, j-1) C(2 n-4-r, n-3-r+j)$. Then it follows that $w_{r}=\sum_{j=1}^{r+2} \gamma_{n, r}(j)\left(e_{j, j}-e_{n+1-j, n+1-j}\right) \in \mathfrak{g}$. After some manipulation it is seen that $w_{0}=C(2 n-4, n-2)\left(e_{1,1}-e_{n, n}+e_{2,2}-e_{n-1, n-1}\right)$. Hence $a=e_{1,1}-e_{n, n}+e_{2,2}-e_{n-1, n-1} \in \mathfrak{g}$. Now $[a, x]=e_{2,3}+e_{n-2, n-1}$. So with the above computation of $\left[z, e_{1,3}-e_{n-2, n}\right]$ we see that $e_{1,2}+e_{n-1, n}$ and $e_{2,3}+e_{n-2, n-1}$ lie in $\mathfrak{g}$.

With $x_{i}$ as above we have

$$
\left[w_{r}, x\right]=\gamma_{n, r}(1) x_{1}+\sum_{j=2}^{r+2} \gamma_{n, r}(j)\left(x_{j}-x_{j-1}\right)
$$

So $\left[w_{r}, x\right]=\delta_{1} x_{1}+\cdots+\delta_{r+1} x_{r+1}+\gamma_{n, r}(r+2) x_{r+2}$, where the $\delta_{i}$ are some coefficients. We have $\gamma_{n, r}(r+2)=-C(2 n-4-r, n-1)$. In general, for $1 \leq i \leq s-1$ we have $C(s, i)=0$ if and only if $s=2 i-1$. Furthermore, $2 n-4-r=2(n-1)-1$ is the same as $r=-1$, which is not the case. So the 
coefficient of $x_{r+2}$ is nonzero. As we have already shown that $x_{1}, x_{2} \in \mathfrak{g}$ it now follows that $x_{i} \in \mathfrak{g}$ for $1 \leq i \leq m$.

Theorem 2.8. Let $\mathfrak{g}$ be a split simple Lie algebra over a field of characteristic 0 . Let $x_{i}, y_{i}, h_{i}$ for $1 \leq i \leq \ell$ be a canonical generating set of $\mathfrak{g}$. Let $y$ be a lowest root vector of $\mathfrak{g}$ and $x=\sum_{i=1}^{\ell} x_{i}$. Let $\mathfrak{a}$ denote the subalgebra generated by $x, y$. Then $\mathfrak{a}$ is simple. More precisely we have

- If $\mathfrak{g}$ is of type $A_{\ell}$ with $\ell$ even then $\mathfrak{a}=\mathfrak{g}$, hence $\mathfrak{a}$ is of type $A_{\ell}$.

- If $\mathfrak{g}$ is of type $A_{\ell}$ with $\ell$ odd then $\mathfrak{a}$ is of type $C_{m}$ with $m=\frac{\ell+1}{2}$.

- If $\mathfrak{g}$ is of type $B_{\ell}, \ell \neq 3$, then $\mathfrak{a}=\mathfrak{g}$, hence $\mathfrak{a}$ is of type $B_{\ell}$.

- If $\mathfrak{g}$ is of type $B_{3}$ then $\mathfrak{a}$ is of type $G_{2}$.

- If $\mathfrak{g}$ is of type $C_{\ell}$ then $\mathfrak{a}=\mathfrak{g}$, hence $\mathfrak{a}$ is of type $C_{\ell}$.

- If $\mathfrak{g}$ is of type $D_{4}$ then $\mathfrak{a}$ is of type $G_{2}$.

- If $\mathfrak{g}$ is of type $D_{\ell}$ with $\ell \geq 5$ then $\mathfrak{a}$ is of type $B_{\ell-1}$.

- If $\mathfrak{g}$ is of type $E_{6}$ then $\mathfrak{a}$ is of type $F_{4}$.

- If $\mathfrak{g}$ if of type $E_{7,8}$ then $\mathfrak{a}=\mathfrak{g}$, hence $\mathfrak{a}$ is of type $E_{7}, E_{8}$ respectively.

- If $\mathfrak{g}$ is of type $F_{4}$ then $\mathfrak{a}=\mathfrak{g}$, hence $\mathfrak{a}$ is of type $F_{4}$.

- If $\mathfrak{g}$ is of type $G_{2}$ then $\mathfrak{a}=\mathfrak{g}$, hence $\mathfrak{a}$ is of type $G_{2}$.

Proof. The statements about the exceptional Lie algebras are easily verified in GAP.

The first, second and fifth points are shown in Proposition 2.5 for a particular canonical generating set. However, two different canonical generating sets are mapped to each other by an automorphism ([15], IV, Theorem 3). Hence these statements hold for any canonical generating set. The third and fourth points are shown similarly, using Proposition 2.7

Suppose that $\mathfrak{g}$ is of type $D_{\ell}$ with $\ell \geq 5$. We consider the diagram automorphism $\phi$ of $\mathfrak{g}$ which fixes $x_{i}, y_{i}, h_{i}$ for $1 \leq i \leq \ell-2$, and interchanges $x_{\ell-1}, x_{\ell}, y_{\ell-1}, y_{\ell}$, $h_{\ell-1}, h_{\ell}$. Then the fixed point subalgebra of $\mathfrak{g}$ with respect to $\phi$ is of type $B_{\ell-1}$. Moreover, $x_{1}, \ldots, x_{\ell-2}, x_{\ell-1}+x_{\ell}, y_{1}, \ldots, y_{\ell-2}, y_{\ell-1}+y_{\ell}, h_{1}, \ldots, h_{\ell-2}, h_{\ell-1}+h_{\ell}$ is a canonical generating set for this algebra. It follows that $x$ is exactly the sum of the positive root vectors in this set. Furthermore, $\phi(y)=y$ and $y$ is a lowest root vector of the fixed point subalgebra. Therefore by the third statement of the proposition it follows that $\mathfrak{a}$ is of type $B_{\ell-1}$.

If $\mathfrak{g}$ is of type $D_{4}$ then we use a similar reasoning. But this time we need to take the diagram automorphism of order 3 (along with the computation for $G_{2}$ ). Alternatively, it can also be established with a straightforward computation in GAP.

\section{FREE DENSE SUbGROUPS OF SIMPLE LIE GROUPS}

In this section we apply generating sets of size 2 of simple Lie algebras obtained in Section 2 to exhibit infinite series of 2-generator free dense subgroups of rank 2 in $\operatorname{SL}(n, \mathbb{C}), \operatorname{Sp}(n, \mathbb{C})$ and in the simple algebraic group of type $G_{2}$ (in its realization as a subgroup of $\operatorname{SL}(7, \mathbb{C})$ ).

As mentioned before, our subgroups are generated by two elements $\exp (t x)$, $\exp (s y)$, where $s, t \in \mathbb{C}$ and $x, y$ are nilpotent elements generating the Lie algebra 
of the group in question. By the following proposition these subgroups are Zariskidense.

Proposition 3.1. Let $G \subset \mathrm{GL}(n, \mathbb{C})$ be a (connected) simple algebraic group with Lie algebra $\mathfrak{g} \subset \mathfrak{g l}(n, \mathbb{C})$. Let $x, y \in \mathfrak{g}$ be nilpotent elements generating $\mathfrak{g}$. Let $t^{\prime}, s^{\prime} \in \mathbb{C}$ be nonzero. Then the group $H=\left\langle\exp \left(t^{\prime} x\right), \exp \left(s^{\prime} y\right)\right\rangle$ is Zariski-dense in $G$.

Proof. Let $\bar{H}$ denote the Zariski-closure of $H$. Then $\bar{H}$ is an algebraic subgroup of $G$ ([4], Proposition 1.3). Let $U=\left\{\exp \left(m t^{\prime} x\right) \mid m \in \mathbb{Z}\right\}$, then $U$ is an infinite subgroup of $H$. Hence $\operatorname{dim} \bar{U} \geq 1$. But $M=\{\exp (t x) \mid t \in \mathbb{C}\}$ is a 1-dimensional algebraic subgroup of $G([4], \S 7.3)$. It follows that $M=\bar{U}$ so that $M \subset \bar{H}$. But then the Lie algebra of $M$ is contained in the Lie algebra of $\bar{H}$. The Lie algebra of $M$ is spanned by $x([4], \S 7.3)$. We see that $x$ lies in the Lie algebra of $\bar{H}$. By the same reasoning the same holds for $y$. It follows that the Lie algebras of $\bar{H}$ and $G$ coincide. So because $G$ is connected, it follows that $\bar{H}=G([4], \S 7.1)$.

We use the well-known ping-pong lemma to show that a group like the group $H$ of the previous proposition is free. For its proof we refer to [18], Proposition 12.2 of Chapter III.

Lemma 3.2 (Ping-Pong Lemma). Suppose a group $G$ acts on a set $X$ and let $H_{1}$ and $\mathrm{H}_{2}$ be subgroups of $G$. Suppose there exist nonempty subsets $A$ and $B$ of $X$ such that

- $B$ is not contained in $A$,

- $h_{1} A \subset B$ for each nonidentity $h_{1} \in H_{1}$,

- $h_{2} B \subset A$ for each nonidentity $h_{2} \in H_{2}$.

Then the group $H=\left\langle H_{1}, H_{2}\right\rangle$ is isomorphic to the free product $H_{1} * H_{2}$ of groups $H_{1}$ and $H_{2}$. In particular, if $H_{1}$ and $H_{2}$ are infinite cyclic groups, $H$ is isomorphic to the free nonabelian group of rank 2.

We adhere to the following notation. Let $x:=\sum_{i=1}^{n-1} e_{i, i+1}, y:=e_{n, 1}$, and $z:=$ $\sum_{i=1}^{n-1} b_{i} e_{i+1, i}$. Then $x, y$ generate $\mathfrak{s l}(n, \mathbb{C})$ for $n$ odd and they generate $\mathfrak{s p}(n, \mathbb{C})$ when $n$ is even (Lemma 2.5). Furthermore, $x, z$ generate many types of algebras for varying choices of the $b_{i}$. For instance, if the $b_{i}$ are as in Example 2.3 then $x, z$ generate $\mathfrak{s l}(n, \mathbb{C})$. 
Their exponentials are

$$
\begin{aligned}
a(t) & :=\exp (t x)=1_{n}+\sum_{i<j} \frac{t^{j-i}}{(j-i) !} e_{i, j}, \\
b(s) & :=\exp (s y)=1_{n}+s e_{n, 1}, \\
c(r) & :=\exp (r z)=1_{n}+\sum_{j=2}^{n} \sum_{i=1}^{j-1} c_{j-i, j} \frac{r^{j-i}}{(j-i) !} e_{j, i} \text { where } c_{j-i, j}=\prod_{k=1}^{j-i} b_{j-k} .
\end{aligned}
$$

We let these act on $\mathbb{C}^{n}$ from the left. This means that we view the elements of $\mathbb{C}^{n}$ as column vectors. However, for reasons of convenience, we write them as row vectors. Let

$$
\begin{aligned}
& X_{1}:=\left\{\left(x_{1}, \ldots, x_{n}\right) \in \mathbb{C}^{n}|| x_{1}|>| x_{i} \mid, 2 \leq i \leq n\right\} \\
& X_{2}:=\left\{\left(x_{1}, \ldots, x_{n}\right) \in \mathbb{C}^{n}|| x_{n}|>| x_{i} \mid, 1 \leq i \leq n-1\right\} .
\end{aligned}
$$

Lemma 3.3. There exists $t_{0} \in \mathbb{R}$ such that $a(t)^{m} X_{2} \subset X_{1}$ for all $t \in \mathbb{C}$ with $|t|>t_{0}$, and $m \in \mathbb{Z}, m \neq 0$.

Proof. Since $a(t)^{m}=a(m t)$ it is enough to show that there is a $t_{0}>0$ such that $a(t) X_{2} \subset X_{1}$ when $|t|>t_{0}$.

Let $x:=\left(x_{1}, \ldots, x_{n}\right) \in \mathbb{C}^{n}$. Then $a(t) x=\left(y_{1}, \ldots, y_{n}\right)$ with

$$
y_{k}=\sum_{i=k}^{n} \frac{t^{i-k}}{(i-k) !} x_{i}
$$

Hence $a(t) x \in X_{1}$ if and only if

$$
\left|\sum_{i=1}^{n} x_{i} \frac{t^{i-1}}{(i-1) !}\right|>\left|\sum_{i=k}^{n} x_{i} \frac{t^{i-k}}{(i-k) !}\right| \text { for } 2 \leq k \leq n .
$$

Suppose $x \in X_{2}$. Then

$$
\left|\sum_{i=1}^{n} x_{i} \frac{t^{i-1}}{(i-1) !}\right| \geq\left|x_{n}\right| \frac{|t|^{n-1}}{(n-1) !}-\sum_{i=1}^{n-1}\left|x_{i}\right| \frac{|t|^{i-1}}{(i-1) !}>\left|x_{n}\right|\left(\frac{|t|^{n-1}}{(n-1) !}-\sum_{i=1}^{n-1} \frac{|t|^{i-1}}{(i-1) !}\right) .
$$

On the other hand,

$$
\left|\sum_{i=k}^{n} x_{i} \frac{t^{i-k}}{(i-k) !}\right| \leq\left|x_{n}\right| \sum_{i=k}^{n} \frac{|t|^{i-k}}{(i-k) !} \leq\left|x_{n}\right| \sum_{i=1}^{n-1} \frac{|t|^{i-1}}{(i-1) !} .
$$

Hence if $t$ satisfies inequality

$$
\frac{|t|^{n-1}}{(n-1) !}-2 \sum_{i=1}^{n-1} \frac{|t|^{i-1}}{(i-1) !}>0
$$

then $a(t) x \in X_{1}$. 
Since the term with the highest exponent of $|t|$ occurs on the left with positive coefficient, there exists a $t_{0}$ such that the inequalities all hold for $|t|>t_{0}$.

Example 3.4. Using (1) we can derive explicit values of $t_{0}$. For example, if $n=$ 2 then (1) is $|t|-2>0$. Hence we can take $t_{0}=2$. If $n=3$ then (1) is $\frac{|t|^{2}}{2}-2(|t|+1)>0$, i.e. we can take $t_{0}=1+\sqrt{3}$. For $n=4$ we have $\frac{|t|^{3}}{3 !}-2\left(\frac{|t|^{2}}{2}+|t|+1\right)>0$, i.e. $|t|^{3}-6|t|^{2}-12|t|-12>0$, so $t_{0}=7.8$ would do.

Lemma 3.5. Set $s_{0}=2$. If $s \in \mathbb{C}$ is such that $|s|>s_{0}$ then $b(s)^{m} X_{1} \subset X_{2}$ for all $m \in \mathbb{Z}, m \neq 0$.

Proof. Similar to the proof of Lemma 3.3 , it is enough to show that $b(s) X_{1} \subset X_{2}$ for $|s|>2$.

Let $x=\left(x_{1}, \ldots, x_{n}\right) \in \mathbb{C}^{n}$. Then

$$
b(s) x=\left(x_{1}, x_{2}, \ldots, x_{n-1}, s x_{1}+x_{n}\right) .
$$

Suppose $x \in X_{1}$, i.e. $\left|x_{1}\right|>\left|x_{i}\right|, 2 \leq i \leq n$. We have

$$
\left|s x_{1}+x_{n}\right| \geq|s|\left|x_{1}\right|-\left|x_{n}\right|>|s|\left|x_{1}\right|-\left|x_{1}\right|=\left|x_{1}\right|(|s|-1) .
$$

Now if $|s|>2$ then $\left|x_{1}\right|(|s|-1)>\left|x_{1}\right|>\left|x_{i}\right|, 2 \leq i \leq n-1$, i.e. $b(s) x \in X_{2}$, as required.

Lemma 3.6. There is an $r_{0}>0$ such that for all $r \in \mathbb{C}$ with $|r|>r_{0}$ we have $c(r)^{m} X_{1} \subset X_{2}$ for all $m \in \mathbb{Z}, m \neq 0$.

Proof. The proof is similar to the one of Lemma3.3, Let $x=\left(x_{1}, \ldots, x_{n}\right) \in \mathbb{C}^{n}$. Then $c(r) x=\left(y_{1}, \ldots, y_{n}\right)$ with

$$
y_{j}=\sum_{i=1}^{j} x_{i} c_{j-i, j} \frac{r^{j-i}}{(j-i) !} .
$$

Suppose that $x \in X_{1}$. Then

$$
\begin{aligned}
\left|\sum_{i=1}^{n} x_{i} c_{n-i, n} \frac{r^{n-i}}{(n-i) !}\right| & \geq\left|x_{1}\right| \frac{|r|^{n-1}}{(n-1) !}\left|c_{n-1, n}\right|-\sum_{i=2}^{n}\left|x_{i}\right| \frac{|r|^{n-i}}{(n-i) !}\left|c_{n-i, n}\right| \\
& \geq\left|x_{1}\right|\left(\frac{|r|^{n-1}}{(n-1) !}\left|c_{n-1, n}\right|-\sum_{i=2}^{n} \frac{|r|^{n-i}}{(n-i) !}\left|c_{n-i, n}\right|\right) .
\end{aligned}
$$

Furthermore,

$$
\left|\sum_{i=1}^{j} x_{i} c_{j-i, j} \frac{r^{j-i}}{(j-i) !}\right| \leq\left|x_{1}\right| \sum_{i=1}^{j}\left|c_{j-i, j}\right| \frac{|r|^{j-i}}{(j-i) !} .
$$

So we require $r$ to satisfy the inequalities

$$
\frac{|r|^{n-1}}{(n-1) !}\left|c_{n-1, n}\right|-\sum_{i=2}^{n} \frac{|r|^{n-i}}{(n-i) !}\left|c_{n-i, n}\right|>\sum_{i=1}^{j}\left|c_{j-i, j}\right| \frac{|r|^{j-i}}{(j-i) !}
$$

for $1 \leq j \leq n-1$. Again we see that there is an $r_{0}$ such that these equalities are satisfied when $r>r_{0}$. 
Example 3.7. Also here we can determine explicit values for $r_{0}$. Let $n=4$, and let $b_{1}, b_{2}, b_{3}$ be as in Example 2.3. Then the inequalities (2) are $224|r|^{3}-84|r|^{2}-$ $14|r|-2>0,224|r|^{3}-84|r|^{2}-22|r|-2>0,224|r|^{3}-132|r|^{2}-26|r|-$ $2>0$. The polynomials on the left-hand side each have one real root, which is approximately, respectively, $0.53,0.57,0.76$. Hence we could take $r_{0}=1$.

Proposition 3.8. Define $H_{n}(t, s):=\langle a(t), b(s)\rangle$. Let $t_{0}$ be as in Lemma 3.3 and $s_{0}$ as in Lemma 3.5. Then for $t, s \in \mathbb{C}$ with $|t|>t_{0},|s|>s_{0}$ we have that $H_{2 m}(t, s)$ and $H_{2 m+1}(t, s)$ are free dense subgroups of rank 2 of $\operatorname{Sp}(2 m, \mathbb{C})$ and $\mathrm{SL}(2 m+1, \mathbb{C})$ respectively.

Proof. In view of Lemmas 3.3, 3.5, the ping-pong lemma (Lemma 3.2) shows that $H_{n}(t, s)$ is free of rank 2. In view of Proposition 2.5, Proposition 3.1 shows that $H_{n}(t, s)$ is dense in $\operatorname{Sp}(n, \mathbb{C})$ if $n$ is even and in $\operatorname{SL}(n, \mathbb{C})$ when $n$ is odd.

Remark 3.9. Proposition 3.8 implies that $H_{2}(t, s)$ is free for $|t|>2,|s|>2$ (see Section 1).

We can consider the group $L_{n}(t, r)$ generated by $a(t), c(r)$ (for various values of the $b_{i}$ ) and use the same arguments (replacing Lemma 3.5 with Lemma 3.6) to get free dense subgroups in various other algebraic groups. For example, with the $b_{i}$ as in Example 2.3 we get free dense subgroups of $\operatorname{SL}(n, \mathbb{C})$ for every $n \geq 3$. The next example makes this precise for $n=4$. Subsequently we have an example showing how in the same way we get a free dense subgroup in the simple algebraic group of type $G_{2}$ (in its representation as a group of $7 \times 7$-matrices).

Example 3.10. Let $n=4$ and $b_{1}=8, b_{2}=12, b_{3}=14$ as in Example 2.3. Consider the $4 \times 4$-matrices

$$
\left(\begin{array}{cccc}
1 & t & \frac{1}{2} t^{2} & \frac{t^{3}}{6} \\
0 & 1 & t & \frac{1}{2} t^{2} \\
0 & 0 & 1 & t \\
0 & 0 & 0 & 1
\end{array}\right),\left(\begin{array}{cccc}
1 & 0 & 0 & 0 \\
8 r & 1 & 0 & 0 \\
48 r^{2} & 12 r & 1 & 0 \\
224 r^{3} & 84 r^{2} & 14 r & 1
\end{array}\right) .
$$

Then if $|t|>8$ and $|r|>1$ these generate a free dense subgroup of $\operatorname{SL}(4, \mathbb{C})$.

Example 3.11. Let $n=7$ and define the following $7 \times 7$-matrices

$$
\begin{aligned}
& x_{1}=e_{23}+e_{56}, y_{1}=e_{32}+e_{65}, h_{1}=\left[x_{1}, y_{1}\right] \\
& x_{2}=e_{12}+e_{34}+e_{45}+e_{67}, y_{2}=e_{21}+2 e_{43}+2 e_{54}+e_{76}, h_{2}=\left[x_{2}, y_{2}\right] .
\end{aligned}
$$

Let $C=\left(\begin{array}{cc}2 & -3 \\ -1 & 2\end{array}\right)$. Then $x_{1}, x_{2}, y_{1}, y_{2}, h_{1}, h_{2}$ satisfy the relations of a canonical set of generators of the Lie algebra of type $G_{2}$ (see Section 2 for the definition of this concept). Hence they generate the Lie algebra of type $G_{2}$. Let $v=(-1,1)$. Then $C v=(-5,3)$. So by Proposition 2.2, $x=x_{1}+x_{2}, z=-y_{1}+y_{2}$ generate the Lie algebra of type $G_{2}$. The matrix $x$ is of the type considered in Lemma 3.3 . The inequality (1) becomes

$$
|t|^{6}-12|t|^{5}-60|t|^{4}-240|t|^{3}-720|t|^{2}-1440|t|-1440>0 .
$$


The polynomial has one positive root, which is approximately 16.6 . So we can take $t_{0}=17$. The matrix $z$ is of the type considered in Lemma 3.6. The inequalities (2) consist of six polynomial inequalities. We will not write down the polynomials here. We found that each of these has a negative and a positive root. The biggest of the positive roots is approximately 16.4. So we can also take $r_{0}=17$.

The conclusion is that if $|t|,|r|>17$ then $\exp (t x), \exp (r z)$ generate a free dense subgroup of the algebraic group of type $G_{2}$.

Corollary 3.12. Let $n>2$ and write $n=2 m$ if $n$ is even, and $n=2 m+1$ if $n$ is odd. Let $t_{0}, s_{0}$ be as in Proposition 3.8 and let $t \in(n-1) ! \mathbb{Z}, s \in \mathbb{Z}$ be such that $|t|>t_{0}$ and $|s|>s_{0}$. Then $H_{2 m+1}(t, s)$ and $H_{2 m}(t, s)$ are thin subgroups of $\mathrm{SL}(2 m+1, \mathbb{Z})$ and $\operatorname{Sp}(2 m, \mathbb{Z})$ respectively.

Proof. The congruence subgroup property implies that a finite index subgroup of $\mathrm{SL}(n, \mathbb{Z}), \operatorname{Sp}(n, \mathbb{Z}), n>2$, contains a principal congruence subgroup, i.e. the kernel $\Gamma_{m}$ of the reduction modulo $m$ homomorphism for some positive integer $m$. However, $\Gamma_{m}$ is not free as has a non-abelian unipotent (i.e. nilpotent) subgroup. Hence, as $H_{n}(t, s)$ is free it is of infinite index.

Similarly, we can construct thin subgroups of $\operatorname{SL}(2 m, \mathbb{Z})$. For example, if $t, r$, $b_{1}, b_{2}, b_{3}$ are as in Example 3.10 then $L_{4}(t, r)<\mathrm{SL}(4, \mathbb{Z})$ is thin for $t \in 6 \mathbb{Z}, r \in \mathbb{Z}$. Notice that in [13] another infinite series of free dense subgroups of $\operatorname{SL}(n, \mathbb{Z})$ were obtained. Those subgroups are generated by $n$ elements and are of rank $n$.

The above results enable us to construct further examples of free dense subgroups. Consider the groups $H_{n}(t, s):=\langle a(t), b(s)\rangle$ as in Proposition 3.8. As we have noted, $a(t)^{k}=a(k t)$ and $b(s)^{k}=b(k s)$ for $k \in \mathbb{Z}$. Now let $S, T$ be indeterminates and consider a word

$$
W(S, T)=a\left(k_{1} T\right) b\left(l_{1} S\right) \cdots a\left(k_{u} T\right) b\left(l_{u} S\right) .
$$

We say that this word is nontrivial if at least one of the $k_{i}, l_{j}$ is nonzero. Observe that $W(S, T)$ is an $n \times n$-matrix with entries in $\mathbb{Q}[S, T]$. As seen in Proposition 3.8, there are $s_{1}, t_{1} \in \mathbb{C}$ such that $H_{n}\left(s_{1}, t_{1}\right)$ is free. This means that if $W(S, T)$ is nontrivial then $W\left(s_{1}, t_{1}\right)$ is not the identity matrix. In particular $W(S, T)$ itself is not the identity matrix. Now let $s_{2}, t_{2} \in \mathbb{C}$ be algebraically independent. Then if $W(S, T)$ is notrivial, $W\left(s_{2}, t_{2}\right)$ is not the identity. It follows that $H_{n}\left(s_{2}, t_{2}\right)$ is free.

With an analogous argument one sees that there is an $s_{3} \in \mathbb{C}$ such that $H_{n}\left(s_{3}, s_{3}\right)$ is free. This implies that $H_{n}\left(s_{4}, s_{4}\right)$ is free if $s_{4}$ is transcendental over $\mathbb{Q}$. In particular, let $s_{4} \in \mathbb{R}$ be transcendental, $\left|s_{4}\right|<1$. Then $H_{n}\left(s_{4}^{k}, s_{4}^{k}\right), k \in \mathbb{Z}, k>0$, gives a family of 2-generator free dense subgroups with generators in an arbitrary small neighborhood of the identity $1_{n}$ ( $\mathrm{cf}[17$, Theorem 7]). Now Theorem 2.1 in [5] implies that for large enough $k$ we have that $H_{n}\left(s_{4}^{k}, s_{4}^{k}\right)$ is free and dense in the Euclidean topology. Furthermore, if $s$ is algebraic and $|\sigma(s)|>\max \left\{s_{0}, t_{0}\right\}$ for some $\sigma \in \operatorname{Gal}(\mathbb{Q}(\mathrm{s}) / \mathbb{Q})$ then $H_{n}(s, s)$ is free.

Similarly, one can construct new families of free subgroups using $L_{n}(t, r)$. 
Acknowledgments. We are grateful to Professors Dmytro Savchuk, Vladimir Shpilrain for fruitful discussions on free groups. We thank the Hausdorff Research Institute for Mathematics for hospitality and facilitation of this research through the Trimester Program 'Logic and Algorithms in Group Theory'. A. S. Detinko was supported by Marie Skłodowska-Curie Individual Fellowship grant H2020 MSCAIF-2015, no. 704910 (EU Framework Programme for Research and Innovation).

\section{REFERENCES}

[1] Richard Aoun. Random subgroups of linear groups are free. Duke Math. J., 160(1):117-173, 2011.

[2] Goulnara Arzhantseva and Arindam Biswas. Large girth graphs with bounded diameter-by-girth ratio. arxiv.org/abs/1803.09229,2018.

[3] A. F. Beardon. Pell's equation and two generator free Möbius groups. Bull. London Math. Soc., 25(6):527-532, 1993.

[4] Armand Borel. Linear algebraic groups, volume 126 of Graduate Texts in Mathematics. Springer-Verlag, New York, second edition, 1991.

[5] E. Breuillard and T. Gelander. On dense free subgroups of Lie groups. J. Algebra, 261(2):448467, 2003.

[6] Alisa Chistopolskaya. On nilpotent generators of the Lie algebra $\mathfrak{s l}_{n}$. Linear Algebra Appl., 559:73-79, 2018.

[7] Willem A. de Graaf. Constructing semisimple subalgebras of semisimple Lie algebras. J. Algebra, 325:416-430, 2011.

[8] A. S. Detinko, D. L. Flannery, and E. A. O'Brien. Algorithms for the Tits alternative and related problems. J. Algebra, 344:397-406, 2011.

[9] D. B. A. Epstein. Almost all subgroups of a Lie group are free. J. Algebra, 19:261-262, 1971.

[10] Elena Fuchs. The ubiquity of thin groups. In Thin groups and superstrong approximation, volume 61 of Math. Sci. Res. Inst. Publ., pages 73-92. Cambridge Univ. Press, Cambridge, 2014.

[11] Elena Fuchs and Igor Rivin. Generic thinness in finitely generated subgroups of $\mathrm{SL}_{n}(\mathbb{Z})$. Int. Math. Res. Not. IMRN, (17):5385-5414, 2017.

[12] The GAP Group. GAP - Groups, Algorithms, and Programming, Version 4.10.0, 2018.

[13] Stephen P. Humphries. Free subgroups of $\mathrm{SL}(n, \mathbf{Z}), n>2$, generated by transvections. $J$. Algebra, 116(1):155-162, 1988.

[14] Tudor Ionescu. On the generators of semisimple Lie algebras. Linear Algebra and Appl., 15(3):271-292, 1976.

[15] Nathan Jacobson. Lie algebras. Interscience Tracts in Pure and Applied Mathematics, No. 10. Interscience Publishers (a division of John Wiley \& Sons), New York-London, 1962.

[16] Victor G. Kac. Infinite-dimensional Lie algebras. Cambridge University Press, Cambridge, third edition, 1990.

[17] Masatake Kuranishi. On everywhere dense imbedding of free groups in Lie groups. Nagoya Math. J., 2:63-71, 1951.

[18] Roger C. Lyndon and Paul E. Schupp. Combinatorial group theory. Springer-Verlag, BerlinNew York, 1977. Ergebnisse der Mathematik und ihrer Grenzgebiete, Band 89.

[19] Peter Sarnak. Notes on thin matrix groups. In Thin groups and superstrong approximation, volume 61 of Math. Sci. Res. Inst. Publ., pages 343-362. Cambridge Univ. Press, Cambridge, 2014.

[20] B. A. F. Wehrfritz. Infinite linear groups. An account of the group-theoretic properties of infinite groups of matrices. Springer-Verlag, New York-Heidelberg, 1973. Ergebnisse der Matematik und ihrer Grenzgebiete, Band 76. 
School of Computer Science, University of St Andrews, United Kingdom

E-mail address: ad271@st-andrews.ac.uk

Dipartimento di Matematica, Università di TREnto, ITALY

E-mail address: degraafascience.unitn.it 